\title{
The telepathology and teleradiology network in Croatia
}

\author{
S. Seiwerth* and Z. Danilovic \\ Institute of Pathology, Medical Faculty, University of \\ Zagreb, Croatia
}

Telepathology as a demanding branch of telemedicine poses a real challenge to experts. The introduction of telepathology in underprivileged countries with poor infrastructure and low health-care budget is a difficult task. On the other hand these countries would mostly benefit by introducing telemedicine/telepathology. In our experience it is possible to build an efficient telepathology/teleradiology network using analogue telephone links and still image transmission, and a store and forward mode of operation. This experience is based on the application of telepathology in Croatia for seven years leading to a national teleradiology network. In this paper the ideas, development and software solutions in the process of establishing a national telepathology and teleradiology network are highlighted.

Keywords: Telepathology, Croatia, teleradiology, still image transmission

\section{Introduction}

The technological level of communication infrastructure (analogue telephone links versus ISDN versus broadband) needed to introduce telemedicine in general and telepathology in particular is a matter of discussion. The some holds true for the feasibility and adequacy of different operation modes (dynamic versus static; interactive versus store and forward) [1,3,6,13$17,22]$.

Croatia with its specific geographical shape and focal distribution of medical facilities seems to be a country that could greatly benefit from the introduction of telemedicine. This idea was greatly fostered by the war 1991-1995. During this time large parts of national territory were under Serb control and numerous vital communication routes were closed. The De-

\footnotetext{
*Corresponding author: Ass. Prof. Sven Seiwerth M.D., Ph.D., Institute of Pathology Medical Faculty University of Zagreb, Salata 10, Croatia. Tel.: +3851 4566 977/980; Fax: +38514683829.
}

partments of pathology equipped with relatively few, sometimes little experienced staff members and some of them working in isolated or hardly accessible hospitals (like Dubrovnik), felt this need very strongly. After some experimental work during 1993, the concept of telepathology in Croatia was launched $[2,4]$. Contemporary to this work, a project (supported by the Ministry of Science) with the scope of telepathology as a basis for Telemedicine started. The technical prerequisites, inherited from disintegrated Yugoslavia, were poor: no other infrastructure except the plain old telephone system (POTS) was available. Most of the hospitals, university hospitals and university buildings had analogue, operator driven switchboards. The development of adequate software seemed to be the next important steps. A system should be developed which would be able to function under the given, rather primitive circumstances. In addition, it should be the adaptable to future technical developments $[2,8,9]$. It became obvious that this task could only be performed in a close cooperation between medical and computer experts working on the spot. To keep scientific enthusiasm in realistic borders, the cooperation of an university staff and a small, profit-based company (VAMS, Zagreb) seemed to be a good solution. These efforts led at the beginning to a telepathology network, and few years later to a national teleradiology network. The aim of this paper is to highlight the concepts and tools of the enterprise.

\section{Concept of telepathology in Croatia}

Being confronted with the unpleasant situation of war, enemy control areas, isolated hospitals, poor infrastructure and critical finances, the idea of introducing a high-technological tool like telemedicine seemed quite ambitious. The first system in use was a derivation of a custom-made image analyzer. The PC-based system had all equipment and tools required for image acquisition and the possibility to store image files. A simple image transmission program, using a 
whiteboard and enabling interactive "chat mode" was added. Voice communication was possible using a separate telephone line. Based on the experiences working with this system a concept to develop a national telepathology network was elaborated. The geographical and socio-political situation was accounted for. The future network was planed hierarchically with regional centers (Osijek, Rijeka and Split) and a national center in Zagreb. A close cooperation with the newly formed Croatian tumor bank was planed. All communication was planed on POTS, using interactive mode with still image transmission. Quite soon a telepathology network including 6 workstations, comprising four teaching and two peripheral hospitals was established [4].

Meanwhile, the government started to build an optical cable network throughout the country, as a basis for introducing ISDN and ATM. It became obvious that the technical basis for telepathology/telemedicine had to be improved and the development of new software solutions had to be considered. The introduction of the Internet as a powerful tool in information exchange created completely new possibilities and opened new fields of investigation [1]. Trough the gathered experience and the new emerging technologies the "store and forward" mode of operation came into focus of our interest, instead of the interactive mode implemented in the beginning of our studies. The need for further development of the whole telepathology software by adding new programs, such as an multifunctional image database, was also obvious.

\section{Software solutions}

To meet our established needs a bi-module telepathology system was created. It consists of a patient database with multimedia functions (ISSA) and a telepathology module (PHAROS) working on a PC under Windows environment [7,9,10].

ISSA is a patient database allowing simultaneous storage of textual (history, status), alphanumerical (laboratory) or images patients' data as well as sound/ voice (Fig. 1). The images can be captured either directly from source (DICOM standard) or by a camera. CT, MR, US, as well as plain radiographs, images of macroscopic specimens and histological slides can be acquired. An additional tool enables the acquisition of live image sequences ("cine loop") [25].

One of the major drawbacks of the classical store and forward telepathology, as established by different authors, is the inability of the receiving side to scan the whole specimen. Thus, the consultant has to relay upon the judgement and choice of the referring pathologist. To solve this drawback, different solutions have been proposed [12,27]. In 1998 we introduced a new tool, called "patchwork" which seems to be very useful in overcoming this problems. It enables semi-automatic image sampling operated interactively or with a motorized stage. To our opinion and experience a continuous control of the focus has to be maintained even when using the motorized stage. The result of the patchwork is an overview image of the whole specimen, composed of full-size, high quality images. These images can be expanded to a full screen size. In this manner the consulting pathologist has the opportunity to select the field of his interest by himself. The patchwork can consist of as many as needed elements. The image generated in this way can be stored as a single file or as a part of the ISSA patient file. The size of a patchwork file composed of 12 images acquired with 6.3 objective and JPEG compressed is about 1.6 MB (Fig. 2). This file size allows easy manipulation and transmission within few minutes [3]. Recently a tool enabling dynamic store and forward telepathology was added to ISSA. The histological slide can be scanned in a standard fashion and the scanning sequence can be recorded ("cine loop"). The images are of high quality and high resolution. From the recorded sequence every single image can be separately analyzed and manipulated (enlarged, stored, transmitted). However, storage of these sequences requires space (5-8 times the space of the corresponding patchwork), and a longer transmission time has to be taken into account [25].

All the pictorial data described above and stored in ISSA can be compressed and sent via ordinary telephone line or Internet, or can be attached to an e-mail. Using this mode the images can be assessed by any potential communication partner. An other possibility is to send the whole ISSA patient file, including textual part, alphanumeric data and images. This type of file can only be opened by a corresponding program on the receiving side. By this point-to-point communication patient data protection is ensured.

The database has also the most often used morphometric operations (distance, counting, perimeter, area) needed in daily routine.

PHAROS is a teletransmission module. It works on the multipage whiteboard principle, with additional chat fields. Single images or patchworks can be placed on the whiteboard. Interactive and store and forward 


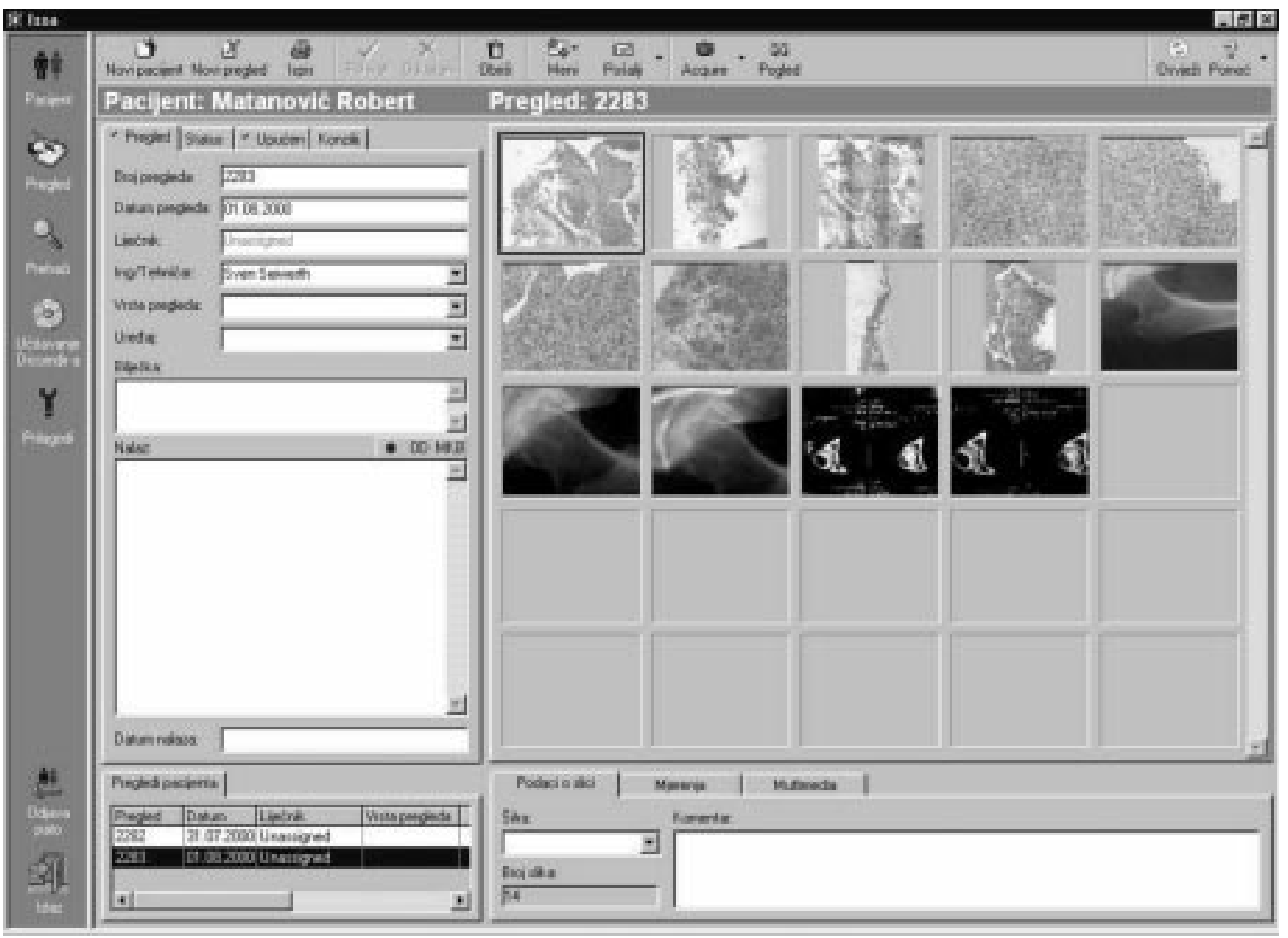

Fig. 1. ISSA patient data file (images from different source).

mode of operation can be used. When using POTS, frozen image transmission is the only possibility. In the interactive mode of operation two cursors and different marking and measuring tools are simultaneously displayed on both communication ends. In the mean time voice communication (trough voice modem) and chat screen are enabled. The program continuously records a communication chart, a feature important due to legal reasons. In the store and forward mode whole ISSA files can be transmitted trough PHAROS using a telephone lines or Internet. During transmission the program ensures that all data are successfully transmitted by continuous checking received against sent data [25].

Despite the existing national telepathology network, most of the routine teleconsultation work is done with international partners, such as K. Kayser (Heidelberg, Germany), L. Banach (Umtata, South Africa) and with the AFIP (Washington DC, USA) (Fig. 3). Internet based, store and forward mode is definitely the most often used way of communication.

\section{From telepathology to teleradiology}

Soon after the first telepathology network has been established and the program solutions described above have been verified in practice, a national teleradiology network has been established in Croatia. In 1998 the network, countrywide linking 33 CT machines in 27 hospitals, has become fully operational. Currently, the system with both of its modules is mostly used for filmless archiving and for remote neurosurgical consultations. The former is saving large amounts of money and storage space while the later saves patients' lives or ensures optimal treatment quality (e.g., by avoiding unnecessary transport or speeding it up when needed) [8]. The neurosurgical consultation center is located at the Neurosurgical Clinic Rebro in Zagreb. More than three hundred consultation cases are handled per year. Most of them are neurotraumata or brain tumors.

The above-described feature of cine loop is used for capturing images from angiographic examinations or 


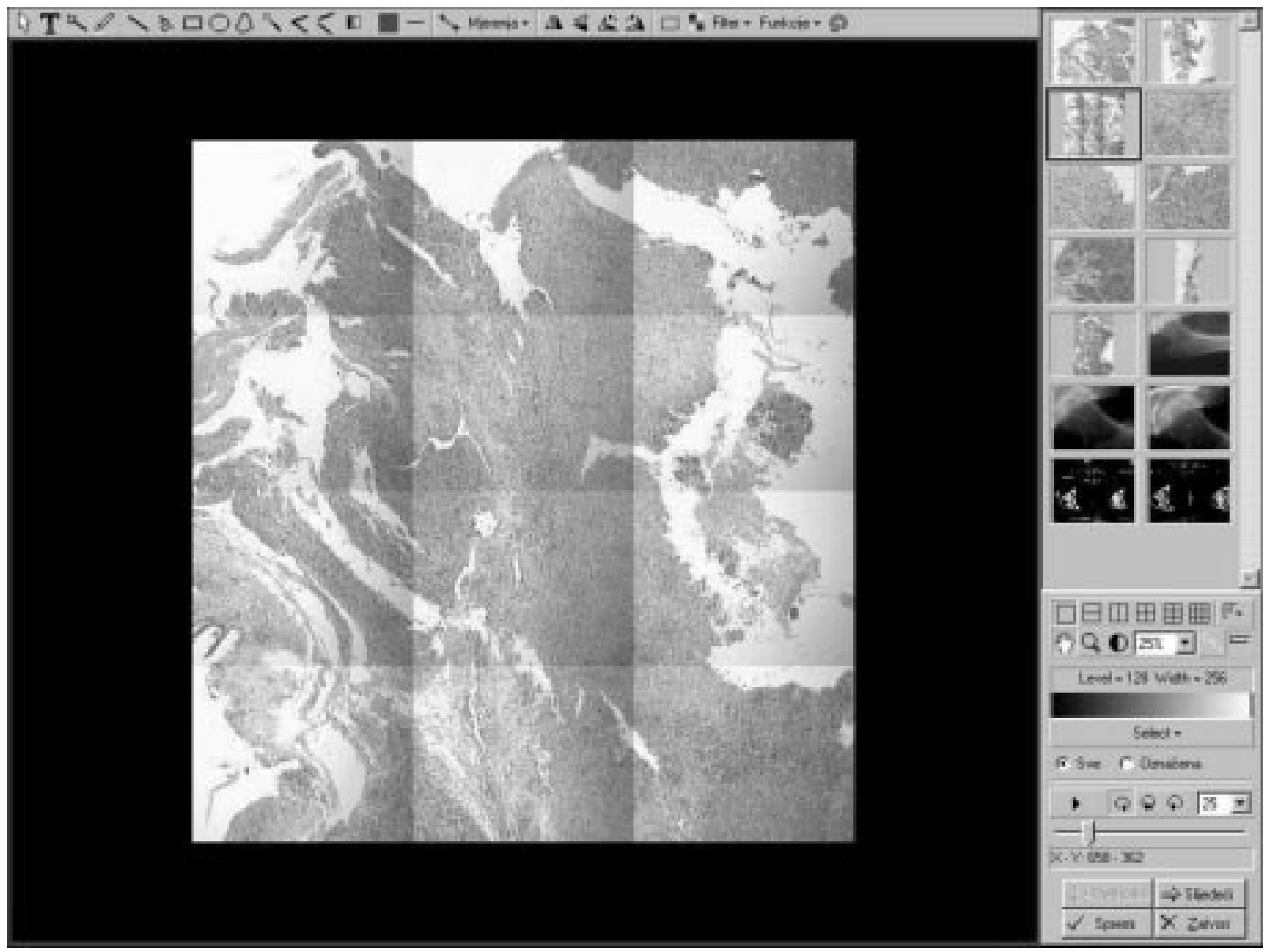

Fig. 2. Patchwork image consisting of 12 full-size high quality images. Each image can be expanded to full screen size.

ultrasound sequences. As these files can be very large. For consultation purposes they can be stored on a CD and sent by overnight delivery or carrier.

\section{The future}

Despite the fact of a functional ATM system in Croatia its use is mainly restricted to universities and companies. Medical institutions supposed to work under economically feasible conditions and with limited finances are not able to take this load. Just recently the introduction of ISDN started very strongly, promising a financially more acceptable burden for the healthcare system.

It is to be expected that in the near future the national teleradiology and telepathology networks will switch to ISDN based communication. As this trend has been expected from the development seen in countries with higher technological standard, the telepathol- ogy/teleradiology system described above has been designed to meet requirements of these more advanced communication channels.

\section{Conclusion}

Many experts in telepathology support the principle that only very complex systems with live image transmission and robotic microscopes based on broad band communication channels can give satisfactory results in routine work [21-27]. In our opinion, systems designed for analogue lines (POTS) can also produce high quality results if adequate software solutions are applied [5,11,18-20,26].

The importance of building simple to operate, high quality systems, which can run on POTS as well as on ISDN or ATM can not be overemphasized. Such systems can introduce telepathology/teleradiology/telemedicine into financially underprivileged countries, 


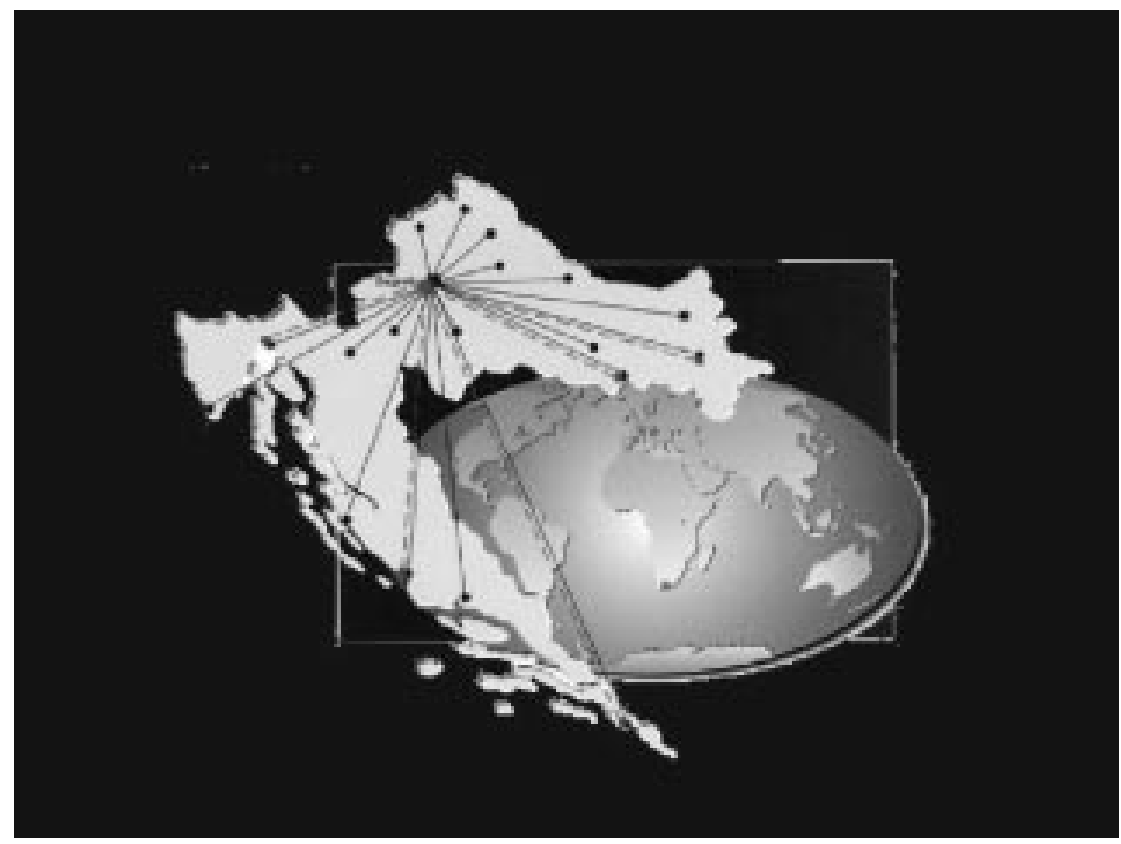

Fig. 3. Telepathology and teleradiology networks in Croatia.

which will greatly benefit from introduction of these new technologies. Integrating patients' databases and telemedicine tools into a singular information system seems also to be of importance. We have the feeling that a system which grew from a relatively small telepathology network to a national teleradiology network in a relatively poor country represents a model which can give useful information about future research and development directions.

\section{References}

[1] C.A. Beltrami and V. Della Mea, Second opinion consultation trough the Internet, Adv. Clin. Path. 2 (1998), 146-148.

[2] Z. Danilovic, A. Dzubur and S. Seiwerth, Concept of telepathology in Croatia, Arch. Anat. Cytol. Pathol. 43 (1995), 282-284.

[3] Z. Danilovic, S. Seiwerth, K. Kayser, L. Banach, D. Babic and A. Dzubur, Experience based approach to interactive versus "store and forward" telepathology, Adv. Clin. Path. 2 (1998), 149-150.

[4] Z. Danilovic and S. Seiwerth, Telepatologija u Hrvatskoj, in: Proceedings Telemedicina u Hrvatskoj, Zagreb, 1998, pp. 181188.

[5] M.N. Doolittle, K.W. Doolittle, Z. Winkelman and D.S. Weinberg, Color images in telepathology: how many colors do we need?, Hum. Pathol. 28 (1997), 36-41.

[6] B.E. Dunn, U.A. Almagro, H. Choi, D.L. Recla and R.S. Weinstein, Use of telepathology for routine surgical pathology review in a test bed in the Department of Veterans Affairs, Telemed. J. 3 (1997), 1-10.
[7] A. Dzubur, Z. Danilovic, N. Caklovic and S. Seiwerth, A contribution to quantitative analysis of transmitted images, Arch. Anat. Cytol. Pathol. 43 (1995), 268-270.

[8] A. Dzubur, International PACS/Teleradiology spotlights: Croatia, Telemedicine Today (December 1998), 35.

[9] A. Dzubur, S. Seiwerth and Z. Danilovic, Benefits of image databank supporting the telepathology system, Adv. Clin. Path. 2 (1998), 158-159.

[10] P. Hufnagl and M. Dietel, The UICC Telepathology Consultation Center, Elec. J. Pathol. Histol. 6(2) (2000), No. 002-12.

[11] K. Kayser, M. Drlicek and W. Rahn, Aids of telepathology in intraoperative histomorphological tumor diagnosis and classification, In vivo 7 (1993), 379-382.

[12] K. Kayser and G. Kayser, Basic aspects of and recent development of telepathology in Europe with specific emphasis on quality assurance, J. Anal. Quant. Cytol. Histol. 21 (1999), 319-328.

[13] K. Kayser and G. Kayser, Telepathology - aspects of social influence and quality control, Elec. J. Pathol. Histol. (1996), No. 963-04.

[14] K. Kayser, G. Kayser and S. Zink, New technical aspects in telepathology, Elec. J. Pathol. Histol. (2000), No. 003-04.

[15] K. Kayser, J. Szymas and R. Weinstein, Telepathology Telecommunication, Electronic Education and Publication in Pathology, Springer, Heidelberg, New York, 1999.

[16] K. Kayser, Telepathology in Europe. Its practical use, Arch. Anat. Cytol. Pathol. 43 (1995), 196-199.

[17] S.Y. Lee and J. Frankel, Ultrasound and other imaging technologies in the intensive care unit, Surg. Clin. North. Am. 80 (2000), 975-1003.

[18] J. Lissner and U. Fink, Digital imaging and picture archiving and communication systems, Curr. Opin. Radiol. 3 (1991), 267-274. 
[19] J. Morrissey, Storing device saves space, money, Mod. Healthc. 25 (1995), 38

[20] S.K. Mun, A.M. Elsayed, W.G. Tohme and Y.C. Wu, Teleradiology/telepathology requirements and implementation, J. Med. Syst. 19 (1995), 153-164.

[21] I. Nordrum, Real-time diagnoses in telepathology, Adv. Clin. Path. 2 (1998), 127-131.

[22] I. Nordrum, A. Amin, V. Isaksen, M. Johansen and J.A. Ludvigsen, Still image consultation via e-mail in surgical pathology, Adv. Clin. Path. 2 (1998), 154-156.

[23] J.M. Schmitz, O. Bollmann and R. Bollmann, Patchwork images as a new technique, Elec. J. Pathol. Histol. 6(2) (2000), No. 002-12.
[24] P. Schwarzmann, B. Binder, R. Klose and M. Kaser, Histkom evaluation of active telepathology in fieldtests, Adv. Clin. Path. 2 (1998), 135-138.

[25] S. Seiwerth, Telepathology and teleradiology, Elec. J. Pathol. Histol. 6(3) (2000), No. 003-06.

[26] Y. Tsuchihashi, T. Mazaki, S. Murata, K. Nakasato, M. Morishima, H. Nagata, I. Tofukuji and K. Naitoh, Telepathology and cytology in Kyoto, Japan to support regional medicine with special references to their need accuracy and cost, Adv. Clin. Path. 2 (1998), 131-132.

[27] R.S. Weinstein, A.K. Bhattacharyya, A.R. Graham and J.R. Davis, Telepathology: a ten year progress report, Hum. Pathol. 28 (1997), 1-7. 


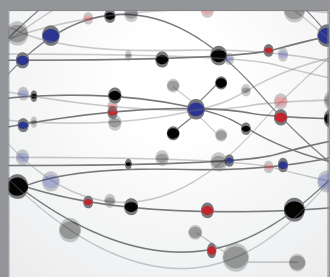

The Scientific World Journal
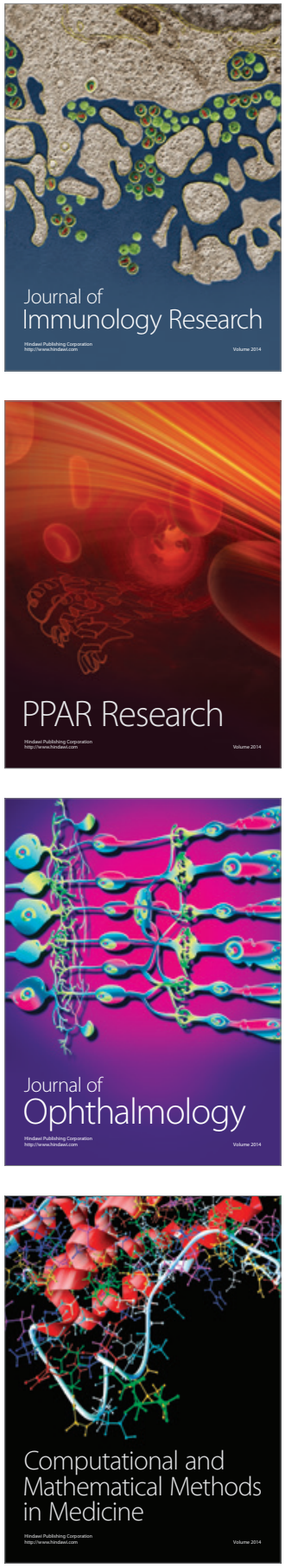

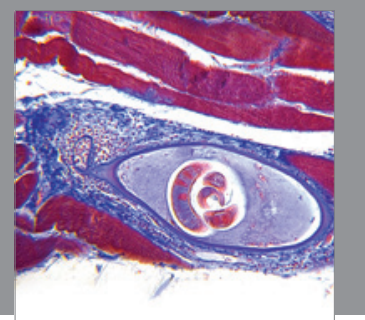

Gastroenterology

Research and Practice
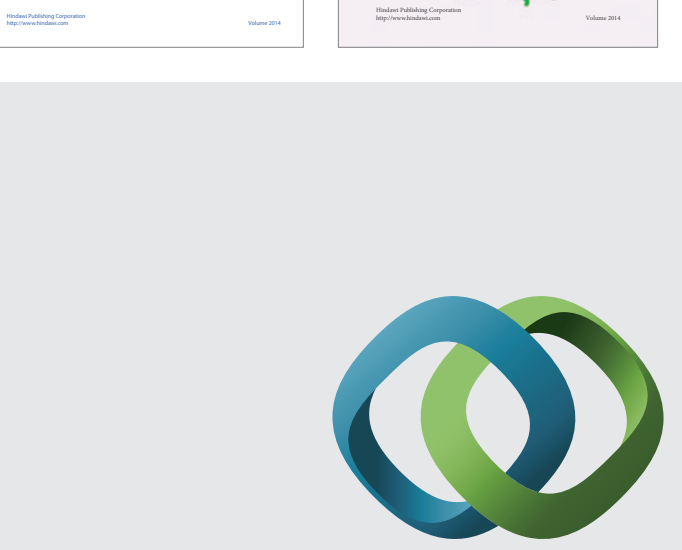

\section{Hindawi}

Submit your manuscripts at

http://www.hindawi.com
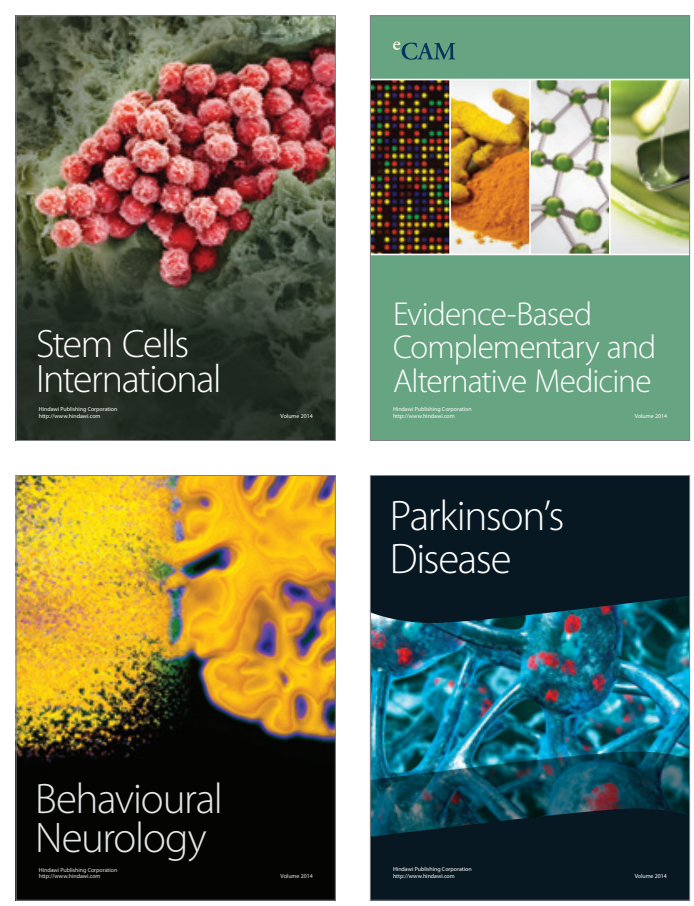

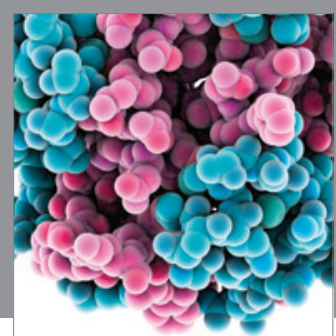

Journal of
Diabetes Research

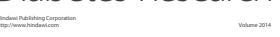

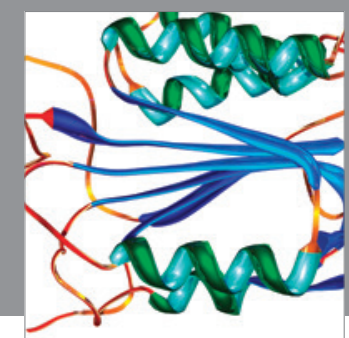

Disease Markers
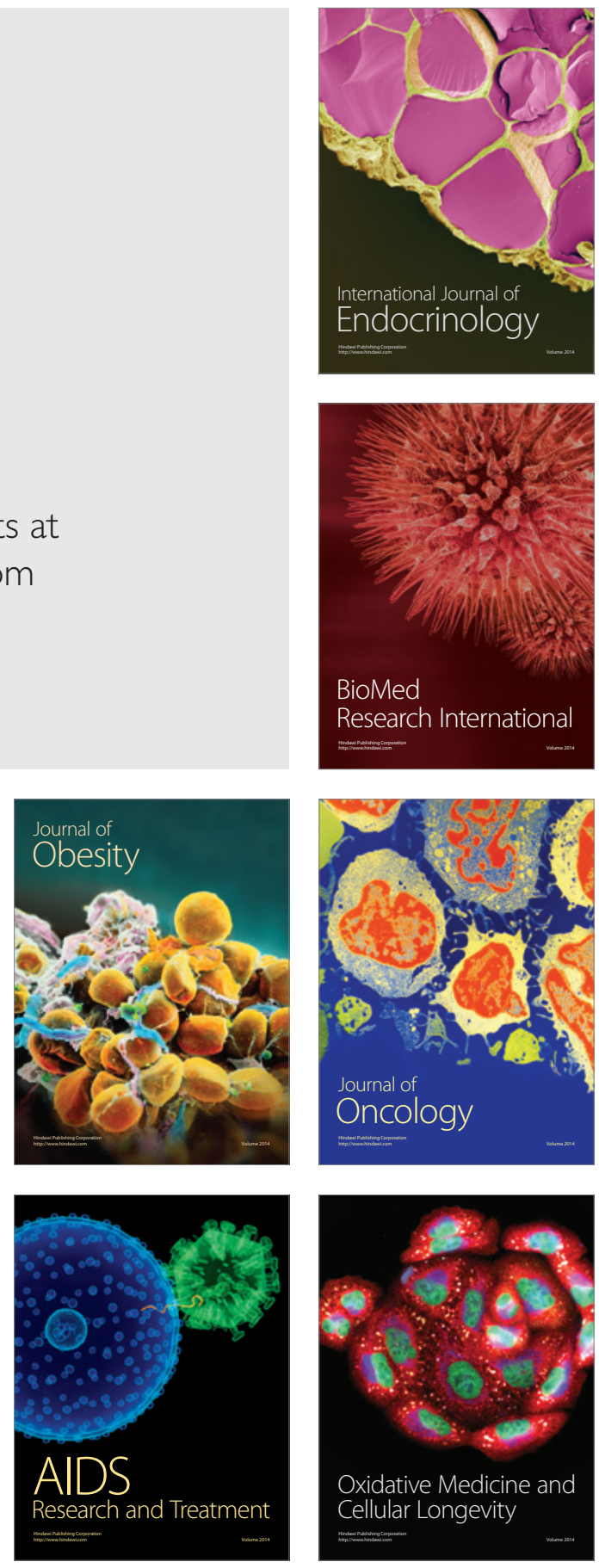